\title{
SOME THEOREMS ON $n$-HOMOGENEOUS CONTINUA
}

\section{E. BURGESS}

1. Introduction and definitions. Bing [2] has given an example of a homogeneous indecomposable continuum in the plane. His example is homeomorphic with an example given by Moise [16] to show the existence of a nondegenerate plane continuum which is not an arc and is homeomorphic with each of its nondegenerate subcontinua. Whyburn [19, Theorem 3] has shown that such a continuum does not separate the plane. Jones [11, Theorem 2] has shown that every homogeneous bounded plane continuum which does not separate the plane is indecomposable. The purpose of this paper is to give some conditions under which a homogeneous continuum is indecomposable and to show that every bounded plane continuum possessing a certain type of homogeneity is a simple closed curve.

If $M$ is a continuum and there exist $n$ continua whose sum is $M$ such that no one of them is a subset of the sum of the others, then $M$ is said to be the finished sum of these $n$ continua.

If the continuum $M$ is the finished sum of $n$ continua and is not the finished sum of $n+1$ continua, then $M$ is said to be indecomposable ${ }^{1}$ under index $n$.

A point set $M$ is said to be $n$-homogeneous if for any $n$ points $x_{1}, x_{2}, \cdots, x_{n}$ of $M$ and any $n$ points $y_{1}, y_{2}, \cdots, y_{n}$ of $M$ there is a homeomorphism $T$ of $M$ onto itself such that $T\left(x_{1}+x_{2}+\cdots+x_{n}\right)$ $=y_{1}+y_{2}+\cdots+y_{n}$. If in addition to these requirements, $T\left(x_{i}\right)=y_{i}$ $(1 \leqq i \leqq n)$, then $M$ is said to be strongly $n$-homogeneous. A point set which is 1 -homogeneous is said to be homogeneous.

A point set $M$ is said to be nearly $n$-homogeneous if for any $n$ points $x_{1}, x_{2}, \cdots, x_{n}$ of $M$ and any $n$ open subsets ${ }^{2} D_{1}, D_{2}, \cdots, D_{n}$ of $M$ there exist $n$ points $y_{1}, y_{2}, \cdots, y_{n}$ of $D_{1}, D_{2}, \cdots, D_{n}$ respectively and a homeomorphism $T$ of $M$ onto itself such that $T\left(x_{1}+x_{2}+\cdots\right.$ $\left.+x_{n}\right)=y_{1}+y_{2}+\cdots+y_{n}$. A set which is nearly 1 -homogeneous is said to be nearly homogeneous.

A point set $M$ is said to be nearly homogeneous over a subset $H$ of $M$ if for any point $x$ of $H$ and any open subset $D$ of $M$ there is a homeomorphism of $M$ onto itself that carries $x$ into a point of $D$.

It is obvious that every $n$-homogeneous set is nearly $n$-homogene-

Presented to the Society, December 29, 1953; received by the editors May 29, 1953.

${ }^{1}$ Some properties of such continua are given in [8] and [18].

2 A subset of $M$ open relative to $M$ is called an open subset of $M$. 
ous and that every nearly $n$-homogeneous set is nearly homogeneous.

2. Homogeneous continua in a metric space. Theorems 1-6 are proved on the basis of R. L. Moore's ${ }^{3}$ Axioms 0 and $1_{3}$. Hence these theorems hold true in any metric space.

TheOREM 1. Every n-homogeneous point set $M$ is homogeneous.

Proof. Suppose $M$ is not homogeneous. Then there are two points $x_{1}$ and $y_{1}$ of $M$ such that there does not exist a homeomorphism of $M$ onto itself that carries $x_{1}$ into $y_{1}$. Let $x_{2}, x_{3}, \cdots, x_{n}$ be distinct points of $M$ different from $x_{1}$ and $y_{1}$. There is a homeomorphism $T$ of $M$ onto itself such that $T\left(x_{1}+x_{2}+\cdots+x_{n}\right)=y_{1}+x_{2}+x_{3}+\cdots+x_{n}$. Since $T\left(x_{1}\right) \neq y_{1}$, then there is a positive integer $i_{1}\left(1<i_{1} \leqq n\right)$ such that $T\left(x_{1}\right)=x_{i_{1}}$. If $T\left(x_{i_{1}}\right) \neq y_{1}$, then there is a positive integer $i_{2}$ $\left(1<i_{2} \leqq n\right)$ different from $i_{1}$ such that $T\left(x_{i_{1}}\right)=x_{i_{2}}$. This process can be continued to obtain a positive integer $r$ such that $1<i_{r} \leqq n$ and $T\left(x_{i_{r}}\right)=y_{1}$. Since $T^{r+1}$ is a homeomorphism of $M$ onto itself carrying $x_{1}$ into $y_{1}$, then $M$ is homogeneous.

THEOREM 2. If $n>1$ and the compact continuum $M$ is indecomposable under index $n$, then $M$ is not nearly homogeneous.

Proof. Suppose, on the contary, that $M$ is nearly homogeneous. By a theorem proved by Swingle [18, Theorem 2], $M$ is the finished sum of $n$ indecomposable continua $M_{1}, M_{2}, \cdots, M_{n}$ such that $M_{1}$ and $M_{2}$ have a point $x$ in common. Let $D$ be an open subset of $M$ which intersects $M_{1}$ and does not intersect $M_{2}+M_{3}+\cdots+M_{n}$. There is a homeomorphism $T$ of $M$ onto itself that carries $x$ into a point of $D$. Then $M$ is the finished sum of the indecomposable continua $T\left(M_{1}\right), T\left(M_{2}\right), \cdots, T\left(M_{n}\right)$. Since $T(x)$ lies in both $T\left(M_{1}\right)$ and $T\left(M_{2}\right)$ and does not lie in $M_{2}+M_{3}+\cdots+M_{n}$, then one of the continua $T\left(M_{1}\right)$ and $T\left(M_{2}\right)$ is not one of the continua $M_{1}, M_{2}, \cdots, M_{n}$. This is contrary to Theorem 2 of [8].

Theorem 3. If $n>1$ and the compact continuum $M$ is nearly homogeneous over a set $H$ consisting of $n$ points about which $M$ is irreducible, then there is a positive integer $r$ not greater than $n$ such that $M$ is indecomposable under index $r$.

Proof. An inductive argument will be used. It will first be shown that Theorem 3 holds true if $n=2$. Let $x$ and $y$ be the two points of $H$. Suppose that $M$ is the finished sum of three continua $M_{1}, M_{2}$, and $M_{3}$. Consider the case in which $x+y$ is a subset of $M_{1}+M_{2}$. Then

${ }^{3}$ Axiom $1_{3}$ is the first three parts of Axiom 1 as stated in [17]. 
$M_{1}+M_{2}$ is not connected and $M_{3}$ intersects each of the continua $M_{1}$ and $M_{2}$. There is a homeomorphism $T$ of $M$ onto itself that carries $x$ into a point of $M-\left(M_{1}+M_{2}\right)$. Then one of the continua $M_{1}+M_{3}$ and $M_{2}+M_{3}$ contains both of the points $T(x)$ and $T(y)$. This is impossible since each of these continua is a proper subset of $M$ and $M$ is irreducible between the points $T(x)$ and $T(y)$. Thus the supposition that $M$ is the finished sum of three continua has led to a contradiction. Hence Theorem 3 holds true if $n=2$.

Now let $k$ be a positive integer such that Theorem 3 holds true if $n=k$. For the remainder of this argument it will be assumed that $H$ consists of $k+1$ points. Suppose that there is a collection $G$ consisting of $k+2$ continua whose finished sum is $M$. Then there is a continuum $X$ of $G$ such that the set $M-(G-X)^{*}$ contains no point of $H$. Let $z$ be a point of $H$. There is a homeomorphism $T$ of $M$ onto itself that carries $z$ into a point of $M-(G-X)^{*}$. Since $T(H)-T(z)$ consists of $k$ points, then some proper subcontinuum $M^{\prime}$ of $M$ contains $T(H)$ $-T(z)$ but not $T(z)$. Since $M^{\prime}+(G-X) *$ is a proper subset of $M$ containing $H$, then $M^{\prime}+(G-X)^{*}$ is not connected. Hence some continuum $L$ of the collection $G-X$ does not intersect $M^{\prime}$. Since $(G-L)^{*}$ is a proper subset of $M$ containing $T(H)$, then $(G-L)^{*}$ is not connected. Hence $(G-L)^{*}$ is the sum of two mutually separated sets $L_{1}$ and $L_{2}$ such that $L_{1}$ contains the continuum $M^{\prime}$. Since $L_{1}+L$ is a proper subcontinuum of $M$, then $L_{2}$ contains $T(z)$. Consequently $L_{2}$ contains the continuum $X$. Since the closed set $(G-X)^{*}$ contains $H$, then $(G-X)^{*}$ is the sum of two mutually separated sets $X_{1}$ and $X_{2}$ such that $X_{1}$ contains the continuum $L_{1}+L$. Hence $X_{1}+X$ contains $T(H)$. This is impossible since $X_{1}+X$ is a proper subcontinuum of $M$ and $M$ is irreducible about $T(H)$. Thus the supposition that $M$ is the finished sum of $k+2$ continua has led to a contradiction. This completes the inductive argument.

THEOREM 4. If the nondegenerate compact continuum $M$ is nearly homogeneous and is irreducible about some finite set, then $M$ is indecomposable.

This theorem follows from Theorems 2 and 3.

COROLLARY. If the nondegenerate compact continuum $M$ is nearly homogeneous and is homeomorphic with each of its nondegenerate subcontinua, then $M$ is indecomposable. ${ }^{5}$

4 The collection of all elements of $G$ different from $X$ is denoted by $G-X$, and the sum of the sets of $G-X$ is denoted by $(G-X)^{*}$.

s If $M$ is a homogeneous plane continuum this follows from [11, Theorem 2] and [19, Theorem 3]. 
THEOREM 5. If $n>1$, then in order that the compact continuum $M$ should be indecomposable under index $n$ it is necessary and sufficient that (1) $M$ should contain a set $H$ consisting of $n$ points such that $M$ is asposyndetic ${ }^{6}$ at each point $x$ of $H$ with respect to each point of $H-x$ and (2) $M$ should not contain $n+1$ such points.

Proof of NeCessity. By [18, Theorem 2], $M$ is the finished sum of $n$ indecomposable continua $M_{1}, M_{2}, \cdots, M_{n}$. Let $H$ be a set consisting of $n$ points $x_{1}, x_{2}, \cdots, x_{n}$ such that for each $i(i \leqq n), x_{i}$ is a point of $M_{i}$ and does not belong to $M_{j}(j \neq i)$. Then $H$ is aposyndetic at each point of $H$ with respect to each other point of $H$. Now suppose that there exists a set $K$ consisting of $n+1$ points of $M$ such that $M$ is aposyndetic at each point of $K$ with respect to each other point of $K$. Then there is a positive integer $i$ such that $M_{i}$ contains two points $y_{1}$ and $y_{2}$ of $K$. There is a continuum $L$ and an open subset $U$ of $M$ such that $M-y_{1} \supset L \supset U \supset y_{2}$. This is contrary to Lemma 1.2 of [7].

Proof of SUfficiency. Since $M$ does not contain $n+1$ points such that $M$ is aposyndetic at each of them with respect to each of the others, then $M$ is not the finished sum of $n+1$ continua. Hence there is a positive integer $r$ not greater than $n$ such that $M$ is indecomposable under index $r$. It follows from the necessary condition proved above that $r=n$.

THEOREM 6. If $n>1$ and the compact continuum $M$ is nearly homogeneous and does not contain $n$ points such that $M$ is aposyndetic at each of them with respect to each of the others, then $M$ is indecomposable.

This theorem follows from Theorems 2 and 5.

3. Homogeneous continua in the plane. The following definition is used in the proof of Theorem 7. A continuum $M$ is said to have property $W$ if every open subset of $M$ contains both a point of a proper subset $K$ of $M$ and a point of $M-K$ such that $K$ is an irreducible cutting ${ }^{7}$ of the plane.

THEOREM 7. If the bounded plane continuum $M$ is nearly homogeneous and separates the plane into a finite number of connected domains, then $M$ is the boundary of each of its complementary domains.

${ }^{6}$ A continuum $M$ is said to be $a p o s y n d e t i c$ at a point $x$ of $M$ with respect to a point $y$ of $M$ if there exists a subcontinuum $K$ of $M$ and an open subset $U$ of $M$ such that $M-y \supset K \supset U \supset x$.

${ }^{7}$ A point set $K$ is said to be an irreducible cutting of the plane if $K$ separates the plane and no proper subset of $K$ does so. Every such set is closed [20, page 43]. 
Lemma 7.1. If the bounded plane continuum $M$ has property $W$ and contains no domain, then every domain intersecting $M$ intersects infinitely many complementary domains of $M$.

Proof of Lemma 7.1. Suppose that this lemma is not true. Then there exists a positive integer $n$ such that (1) every domain intersecting $M$ intersects at least $n$ complementary domains of $M$ and (2) some domain $D$ intersects $M$ and does not intersect $n+1$ complementary domains of $M$. There is a proper subset $K$ of $M$ such that $D$ intersects both $K$ and $M-K$ and $K$ is an irreducible cutting of the plane. There is a connected domain $D^{\prime}$ lying in $D$ and intersecting $M$ but not $K$. There is a complementary domain $E$ of $K$ intersecting $D$ but not $D^{\prime}$. Hence some complementary domain $E^{\prime}$ of $M$ lies in $E$ and intersects $D$ but not $D^{\prime}$. Since $D^{\prime}$ intersects $n$ complementary domains of $M$, then $D$ intersects $n+1$ complementary domains of $M$. This involves a contradiction.

Proof of Theorem 7. Suppose that some complementary domain of $M$ does not have $M$ as its boundary. Since the boundary of this domain separates the plane, it follows from two theorems proved by Kuratowski [13, Theorems 2 and 4] that some proper subcontinuum $K$ of $M$ is an irreducible cutting of the plane. Let $D$ be any open subset of $M$ and let $x$ be a limit point of $M-K$ lying in $K$. There is a homeomorphism $T$ of $M$ onto itself that carries $x$ into a point of $D$. It follows from a theorem proved by Brouwer [5, p. 425] that $T(K)$ is an irreducible cutting of the plane. Since $T(K)$ is a proper subset of $M$ and $T(x)$ is a limit point of $M-T(K)$, it follows that $M$ has property $W$. Since a plane domain is invariant under a homeomorphism [4], it follows that $M$ contains no domain. Hence by Lemma 7.1, every domain intersecting $M$ intersects infinitely many complementary domains of $M$. This is a contradiction since $M$ has only a finite number of complementary domains.

THEOREм 8. If the bounded plane continuum $M$ is nearly homogeneous and has more than two but only a finite number of complementary domains, then $M$ is indecomposable. ${ }^{8}$

Proof. Suppose that $M$ is decomposable. By Theorem 7, $M$ is the boundary of each of its complementary domains. It follows from an argument given by Kuratowski that $M$ is indecomposable under index 2. (See [14, Theorem 3] and the argument thereof.) This is contrary to Theorem 2.

\footnotetext{
8 The author does not know whether there exists a continuum which satisfies the hypothesis of this theorem.
} 
THEOREM 9. If $n>1$ and the bounded plane continuum $M$ is nearly $n$-homogeneous and is not a continuous curve, then $M$ is indecomposable?

InDICATION OF PROOF. Suppose that $M$ is the finished sum of five continua $M_{1}, M_{2}, \cdots, M_{5}$. Let $K_{1}, K_{2}, \cdots, K_{5}$ be circular domains such that (1) $\bar{K}_{1}, \bar{K}_{2}, \cdots, \bar{K}_{5}$ are mutually exclusive, (2) for each $i(i \leqq 5), K_{i}$ intersects $M_{i}$, and (3) if $j \neq i, \bar{K}_{i}$ does not intersect $M_{j}$. By [17, Theorem 47, p. 132], there exist two open subsets $U_{1}$ and $U_{2}$ of $M$, a continuum $H$, and a sequence of mutually exclusive continua $H_{1}, H_{2}, H_{3}, \cdots$ such that (1) $U_{1}$ and $U_{2}$ are mutually exclusive, (2) for each $n, H_{n}$ intersects both $U_{1}$ and $U_{2}$ and is a component of $M-\left(U_{1}+U_{2}\right)$, and (3) the sequence $H_{1}, H_{2}, H_{3}, \cdots$ converges to $H$. Since $M$ is nearly $n$-homogeneous, there exists a homeomorphism $T$ of $M$ onto itself that carries some point $x_{1}$ of $H$ into a point of $K_{1}$ and some point $x_{2}$ of $H$ into a point of $K_{2}$. There exist open subsets $R_{1}$ and $R_{2}$ of $M$ containing $x_{1}$ and $x_{2}$ respectively such that $T\left(R_{1}\right) \subset K_{1}$ and $T\left(R_{2}\right) \subset K_{2}$. Since infinitely many of the sets $H_{1}, H_{2}, H_{3}, \cdots$ intersect both $R_{1}$ and $R_{2}$, then infinitely many of the sets $T\left(H_{1}\right)$, $T\left(H_{2}\right), T\left(H_{3}\right), \cdots$ intersect both $K_{1}$ and $K_{2}$. Consequently there exists an infinite sequence $H_{1}^{\prime}, H_{2}^{\prime}, H_{3}^{\prime}, \cdots$ of mutually exclusive subcontinua of $M$ such that (1) for each $n, H_{n}^{\prime}$ intersects both $\bar{K}_{1}$ and $\bar{K}_{2}$ and is a component of $M-M \cdot\left(K_{1}+K_{2}\right)$, and (2) for each $i$ ( $i \leqq 2)$, some arc $p_{i} q_{i}$ on the boundary $J_{i}$ of $K_{i}$ contains the sets $J_{i} \cdot H_{1}^{\prime}, J_{i} \cdot H_{2}^{\prime}, J_{i} \cdot H_{3}^{\prime}, \cdots$ in the order named from $p_{i}$ to $q_{i}$. For each $n$, there is an arc $a_{n} b_{n}$ irreducible from $\bar{K}_{1}$ to $\bar{K}_{2}$ such that (1) $a_{n}$ is between $J_{1} \cdot H_{n}^{\prime}$ and $J_{1} \cdot H_{n+1}^{\prime}$ on $p_{1} q_{1}$ and $b_{n}$ is between $J_{2} \cdot H_{n}^{\prime}$ and $J_{2} \cdot H_{n+1}^{\prime}$ on $p_{2} q_{2}$ and (2) $a_{n} b_{n}$ does not intersect $M$. For each $n$, let $D_{n}$ denote the complementary domain of $M+\bar{K}_{1}+\bar{K}_{2}$ which contains $a_{n} b_{n}-\left(a_{n}+b_{n}\right)$. Since the domains $D_{1}, D_{2}, D_{3}, \cdots$ are mutually exclusive, it can be shown by an argument quite similar to the one used in Case 1 of the proof of Theorem 9 of [6] that no one of the sets $\bar{K}_{3}, \bar{K}_{4}$, and $\bar{K}_{5}$ intersects more than two of these domains. Hence one of the domains $D_{1}, D_{2}, D_{3}, \cdots$ does not intersect $\bar{K}_{3}+\bar{K}_{4}+\bar{K}_{5}$. From this it follows that if $i \neq j(i, j \leqq 5)$, there is a complementary domain $Z$ of $M+\bar{K}_{i}+\bar{K}_{j}$ and an arc $t$ not intersecting $M$ such that (1) $Z$ does not intersect any of the sets $\bar{K}_{1}, \bar{K}_{2}, \cdots, \bar{K}_{5}$ and (2) $t$ is irreducible from $\bar{K}_{i}$ to $\bar{K}_{j}$ and lies, except for its end points, in $Z$. Let $Z_{1}, Z_{2}, \cdots, Z_{10}$ be ten such domains corresponding to the ten ways the integers $i$ and $j$ can be chosen. An argument similar to the one used in the proof of Theorem 5 of [7] can be used to show that some two of these ten domains have a point in common. Consider the case in which (1) $Z_{1}$ and $Z_{2}$ have a point in common," (2) $Z_{1}$ is a comple-

\footnotetext{
${ }^{\ominus}$ Bing $[2$, Theorem 14] has shown that such a continuum exists.
} 
mentary domain of $M+\bar{K}_{1}+\bar{K}_{2}$ and for each $i(i \leqq 2), \bar{K}_{i}-M \cdot \bar{K}^{i}$ contains a boundary point of $Z_{1}$, and (3) $Z_{2}$ is a complementary domain of $M+\bar{K}_{3}+\bar{K}_{r}$, where $r \neq 3$, and for each $i(i=3, r), \bar{K}_{i}-M$ $\cdot \bar{K}_{i}$ contains a boundary point of $Z_{2}$. Then $Z_{1}$ contains $Z_{2}$ together with every boundary point of $Z_{2}$ that belongs to $\bar{K}_{3}-M \cdot \bar{K}_{3}$. Hence $Z_{1}$ intersects $\bar{K}_{3}$. As this involves a contradiction, it follows that there is a positive integer $k$ less than five such that $M$ is indecomposable under index $k$. Since $M$ is nearly homogeneous, then by Theorem 2 , $M$ is indecomposable.

Theorem 10. If $n>1$ and the bounded plane continuum $M$ is $n$ homogeneous, then $M$ is a simple closed curve.

Proof. Suppose that $M$ is indecomposable. Let $x_{1}, x_{2}, \cdots, x_{n}$ be $n$ points lying in the same composant of $M$ and let $y_{1}, y_{2}, \cdots, y_{n}$ be $n$ points of $M$ such that no composant of $M$ contains all of them. Since some proper subcontinuum of $M$ contains $x_{1}+x_{2}+\cdots+x_{n}$ and no proper subcontinuum of $M$ contains $y_{1}+y_{2}+\cdots+y_{n}$, there does not exist a homeomorphism of $M$ onto itself that carries $x_{1}+x_{2}$ $+\cdots+x_{n}$ into $y_{1}+y_{2}+\cdots+y_{n}$. As this involves a contradiction, then $M$ is decomposable. Since $M$ is nearly $n$-homogeneous, it follows from Theorem 9 that $M$ is a continuous curve. By Theorem 1, $M$ is homogeneous. Mazurkiewicz [15] has shown that every homogeneous bounded plane continuous curve is a simple closed curve. Hence $M$ is a simple closed curve.

4. Questions and remarks. So far as the author knows, the following two questions have not as yet been answered. (1) Does there exist a homogeneous bounded plane continuum which separates the plane and is not a simple closed curve? (See $[12$, p. 149] and $[3, \mathrm{p}$. 49].) Cohen [9] has shown that if such a continuum exists, then it does not contain a simple closed curve. (2) Is every homogeneous decomposable bounded plane continuum $M$ a simple closed curve? Jones [10] has shown that the answer is in the affirmative if $M$ is aposyndetic.

There does exist a nearly homogeneous bounded plane dendron. Such a set can be easily constructed. The set of all end points of any such dendron $M$ would be everywhere dense in $M$.

There exists a decomposable continuum which satisfies the hypothesis of Theorem 3. For each $i(i \leqq 2)$, let $H_{i}$ be a set indicated by Fig. 1 of [6] such that the common part of $H_{1}$ and $H_{2}$ is the point $O$. Let $K_{i}$ be a composant of $H_{i}$ not containing $O$ and let $x_{i}$ be a point of $K_{i}$. The decomposable continuum $H_{1}+H_{2}$ is irreducible between $x_{1}$ and $x_{2}$ and is nearly homogeneous over $x_{1}+x_{2}$. 
Anderson [1] has shown that there exists a one-dimensional compact continuous curve $M$ such that for each $n, M$ is strongly $n$-homogeneous. If $n>3$, then no simple closed curve is strongly $n$-homogeneous. Hence it follows from Theorem 10 that if $n>3$, then no bounded plane continuum is strongly $n$-homogeneous.

\section{BIBLIOGRAPHY}

1. R. D. Anderson, A homogeneous one-dimensional compact continuous curve, Bull. Amer. Math. Soc. Abstract 59-2-249.

2. R. H. Bing, A homogeneous indecomposable plane continuum, Duke Math. J. vol. 15 (1948) pp. 729-742.

3. - Concerning hereditarily indecomposable continua, Pacific Journal of Mathematics vol. 1 (1951) pp. 43-51.

4. L. E. J. Brouwer, Beweis der Invarianz des n-dimensionalen Gebiets, Math. Ann. vol. 71 (1912) pp. 305-313.

5. - Beweis der Invarianz der geschlossenen Kurve, Math. Ann. vol. 72 (1912) pp. 422-425.

6. C. E. Burgess, Continua and their complementary domains in the plane, Duke Math. J. vol. 18 (1951) pp. 901-917.

7. - Continua and their complementary domains in the plane. II, Duke Math. J. vol. 19 (1952) pp. 223-230.

8. - Continua which are the sum of a finite number of indecomposable continua, Proc. Amer. Math. Soc. vol. 4 (1953) pp. 234-239.

9. Herman J. Cohen, Some results concerning homogeneous plane continua, Duke Math. J. vol. 18 (1951) pp. 467-474.

10. F. Burton Jones, $A$ note on homogeneous plane continua, Bull. Amer. Math. Soc. vol. 55 (1949) pp. 113-114.

11. - Certain homogeneous unicoherent indecomposable continua, Proc. Amer. Math. Soc. vol. 2 (1951) pp. 855-859.

12. - Concerning aposyndetic and non-aposyndetic continua, Bull. Amer. Math. Soc. vol. 58 (1952) pp. 137-151.

13. Casimir Kuratowski, Sur les coupures irreductibles du plan, Fund. Math. vol. 6 (1924) pp. 130-145.

14. - Sur la structure des frontières communes à deux régions, Fund. Math. vol. 12 (1928) pp. 20-42.

15. Stefan Mazurkiewicz, Sur les continus homogènes, Fund. Math. vol. 5 (1924) pp. 137-146.

16. E. E. Moise, An indecomposable plane continuum which is homeomorphic to each of its non-degenerate subcontinua, Trans. Amer. Math. Soc. vol. 63 (1948) pp. 581-594.

17. R. L. Moore, Foundations of point set theory, Amer. Math. Soc. Colloquium Publications, vol. 13, 1932.

18. P. M. Swingle, Generalized indecomposable continua, Amer. J. Math. vol. 52 (1930) pp. 647-658.

19. G. T. Whyburn, A continuum every subcontinuum of which separates the plane, Amer. J. Math. vol. 52 (1930) pp. 319-330.

20. —, Analytic topology, Amer. Math. Soc. Colloquium Publications, vol. 28, 1942.

The University of Utah 